Published in: Finite Elements in Analysis and Design, 73C, 42-54.

DOI: http://dx.doi.org/10.1016/j.finel.2013.05.004

\title{
On the relationship between mesh and stress field orientations in linear stability analyses of thin plates and shells
}

\author{
Adam J. Sadowski ${ }^{1} \&$ J. Michael Rotter ${ }^{2}$
}

\begin{abstract}
Many problems in structural mechanics involve complex principal stress fields that are not orthogonal to the geometric axis of the structure. Such problems are often analysed with finite elements, but the quality of a finite element solution may be sensitive to the orientation of the mesh with respect to the principal axes of stress. This paper presents the outline of a procedure to generate well-structured inclined quadrilateral finite element meshes for the analysis of thin plate and shell structures. The procedure was developed using the commercial FE pre-processor ABAQUS CAE and the Python script language, though it may readily be applied in any pre-processor which supports an external scripting functionality.
\end{abstract}

A set of mesh convergence studies using linear buckling analyses are presented on four benchmark problems with known analytical solutions to illustrate the effect of inclined meshes on the accuracy of the computed solution. These illustrations are intended to raise an awareness of the subtle but important relationship between mesh and stress field orientation and are presented for the benefit of practising finite element analysts in structural engineering.

\section{Keywords}

Finite element analysis, mesh orientation, plate buckling, shell buckling, orthogonal helices, helical meshing, linear bifurcation analysis.

${ }^{1}$ Lecturer, Imperial College London, UK

${ }^{2}$ Professor of Civil Engineering, The University of Edinburgh, UK 
Published in: Finite Elements in Analysis and Design, 73C, 42-54.

DOI: http://dx.doi.org/10.1016/j.finel.2013.05.004

\section{Introduction}

The behaviour of plate and shell structures is widely recognised to be among the most complex of all classical structural forms. This complexity originates primarily from the tendency of such structures to buckle into a deformed shape at a load that is highly sensitive to many aspects of the structure and thus difficult to predict with certainty. Unsurprisingly, finite element modelling of these structures comes with many associated challenges and pitfalls for the unwary [1,2,3], a few of which are illustrated in this paper.
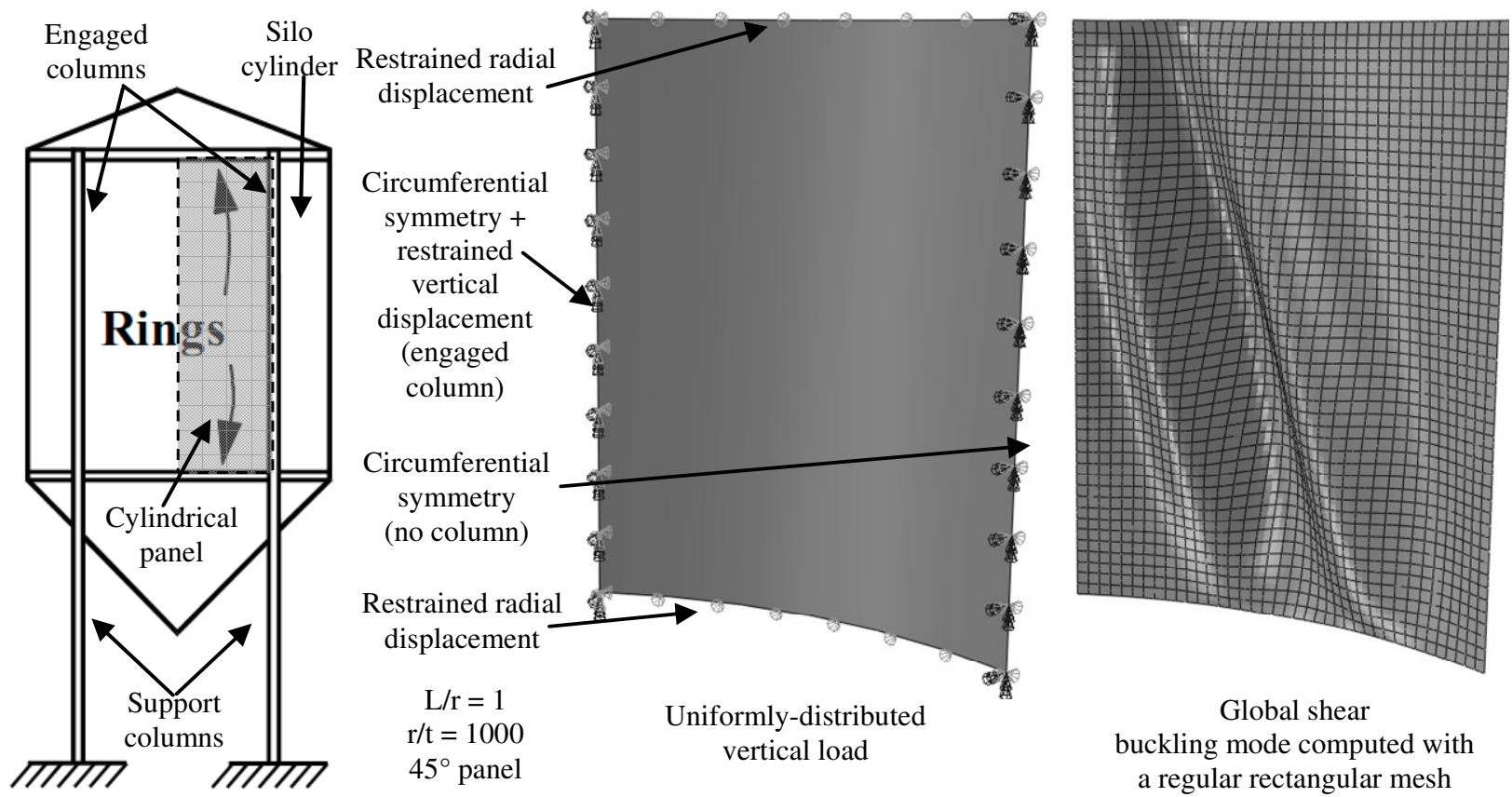

Fig. 1 - Illustration of a shear buckle in a regular mesh of rectangular elements

The buckling of beams and columns mostly involves relatively simple uniaxial stress states. By contrast, many practical problems in plate and shell buckling involve complex three-dimensional stress patterns and localised stress conditions. For example, the buckling mode of plates and shells that are essentially rectangular in form may be in shear at a location associated with a local load introduction or a boundary feature such as a support, stiffener, hole or penetration. This is illustrated qualitatively in Fig. 1 with a simple finite element model showing the typical buckling behaviour of a $45^{\circ}$ cylindrical panel in a silo with engaged columns. The downward action of the friction between the stored granular solid and the silo wall is represented as a uniformly distributed vertical load acting on the surface of the panel, which subjects it to global shear. The finite element mesh consists of rectangular shell elements that are 
Published in: Finite Elements in Analysis and Design, 73C, 42-54.

DOI: http://dx.doi.org/10.1016/j.finel.2013.05.004

orthogonal to the geometric axes of the panel, which is the natural way of modelling a structure of this kind. It is clear that the resulting global shear buckling mode is caused by principal stresses that are not aligned with the axes of the geometry and the mesh. This situation provokes the question of whether a rectangular mesh of elements that are aligned with the principal axes of stress systematically produces more accurate descriptions of the same behaviour. The answer naturally depends on the shape functions assumed in the formulation of the finite element.

The issue raised in the above illustration is relevant to many situations where a shell structure is subject to unsymmetrical load patterns which inevitably result in local shear stress fields. These include, but are not limited to, cylindrical shells under meridional 'strips' of load [4,5,6,7], cylindrical silos and conical hoppers under eccentric discharge $[8,9,10,11]$, cylindrical silos under wind pressures $[12,13]$, silos on bracket supports [14], cylindrical tanks subject to differential settlement [15] and axially-compressed cylinders with cut-outs [16]. Additionally, global shear buckling modes arise in the modelling of cylindrical tanks subject to transverse shear $[17,18]$ and torsion $[19,20]$ which can arise under seismic action. These are but a small portion of the publications available on each respective topic. However, in each of those mentioned here, the authors modelled their respective structures using regular meshes of rectangular elements in the style of Fig. 1 and no mention is made of whether this mesh orientation is indeed the optimal one for the given context.

Methods of adaptive meshing and the effects of mesh anisotropy are intensive research areas in mechanics. The majority of the effort appears to focus on the field of computational fluid dynamics where the modelling of turbulence is particularly computationally intensive and mesh optimisation is crucial [21,22,23]. A number of recent studies have suggested that finite elements often have a 'preferred' net orientation with regard to the contours of a particular field variable. For example, D'Azevedo [24] found that changing the orientation of grids of first order triangular and quadrilateral elements may yield an order of magnitude improvement in accuracy when interpolating convex functions. Troyani et al. [25] found that the orientation of a mesh of regular triangular elements has a significant effect on the solution accuracy of solid sections under torsion and on the magnitude of the calculated shear stresses. Hamide et al. [26] modelled arc welding on a steel plate using a thermo-mechanical 
Published in: Finite Elements in Analysis and Design, 73C, 42-54.

DOI: http://dx.doi.org/10.1016/j.finel.2013.05.004

analysis with adaptive meshing and found that triangular elements become stretched orthogonally to directions of steep temperature gradients while El khaoulani and Bouchard [27] applied an automatic mesh adaptation method to model cracking in tensile tests of aluminium rods and similarly found that triangular elements eventually rearrange to become stretched orthogonally to directions of steep strain gradient. Both of the preceding cases effectively lead to an overall mesh rotation. Unfortunately, adaptive meshing algorithms are typically complex to implement using commercial software, often requiring an a posteriori estimate of the global error, and are beyond the expertise of most structural designers. Also, adaptive meshing is usually limited to triangular elements which are relatively insensitive to further distortion due to changes in nodal coordinates, unlike quadrilateral elements which perform optimally when their geometries are close to square and are widely favoured for structural buckling analyses.

In structural and solid mechanics, the meshing algorithm in a typical finite element pre-processor generates quadrilateral elements that, by default, conform to the primary axes of the geometry of the structure. This usually results in a speedily-generated and well-conditioned regular mesh suitable for the analysis of most classical problems in structural engineering. Irregular geometries tend to be filled in with numericallyinferior but geometrically more versatile triangular elements, typically using Delaunay triangulation algorithms (e.g. [28,29]). As hypothesised above, the quality of predictions in certain problems with relatively simple geometries could benefit from a non-standard mesh orientation of regular quadrilateral elements, especially if it places the axes of the element in a more favourable orientation relative to the principal axes of stress. Additionally, such 'inclined' meshes may even enable the use of regular quadrilateral elements to model more complex geometries which would otherwise need to be meshed freely with lower-order triangular elements. This is especially important in nonlinear stability analyses in structural engineering where much is gained in terms of accuracy by using quadrilateral and higher-order elements [30,31].

This paper presents an outline of a procedure for inclined meshing and illustrates its effect on two simple geometries: a 2D plate and a 3D cylindrical shell. The numerical consequences of inclined meshes of first order elements are subsequently explored through mesh convergence studies on four classical linear bifurcation problems taken 
Published in: Finite Elements in Analysis and Design, 73C, 42-54.

DOI: http://dx.doi.org/10.1016/j.finel.2013.05.004

from the elastic stability theory of thin plates and shells. These four benchmark problems involve a rectangular plate under uniform compression and under uniform shear, and a cylinder under uniform axial compression and under uniform torsion. The method of mesh generation suggested here may be generalised to other geometries and element types, such as 3D solid continuum elements, or even to corresponding problems involving thermal, electric or potential fields. Further, although the meshing procedure is illustrated using the ABAQUS CAE [31] pre-processor, it may be readily implemented in an analogous manner in any other software that supports an external scripting functionality (e.g. ANSYS, FEMAP/NASTRAN, COMSOL etc.).

\section{Inclined meshing with shell elements}

\subsection{Thin 2D rectangular plate}

The task of generating an inclined mesh in a finite element pre-processor is not trivial. For a rectangular 2D geometry, the meshing algorithm will automatically generate a mesh of quadrilateral shell elements that are orthogonal to the axes of the rectangle. If an inclined mesh is desired, it is necessary to begin by partitioning the rectangle into an inclined orthogonal grid (Fig. 2) to force the meshing algorithm to conform to the axes of this grid rather to that of the original rectangle. This type of partitioning may be undertaken manually within a commercial finite element pre-processor, but it is less tedious when the process is automated with appropriate use of scripting. Scripting in ABAQUS is done with the Python object-based programming language, whereas ANSYS and COMSOL use APDL and Matlab-like scripting respectively.

The partitioning procedure is initiated by generating corresponding pairs of horizontal and vertical reference points in the plane of the rectangular surface, as illustrated in Fig. 2a. The line joining each corresponding pair of points forms an angle $\alpha$ with the horizontal axis. The reference points need not be physically on the surface of the rectangle that is to be meshed, but they should be positioned so that lines joining any ordered pair of reference points dissect the rectangle entirely. The full partition grid should then be generated gradually line by line (Figs $2 b \& 2 c$ ). The user should take care in the script to update the geometry of the rectangular surface after every such partition and assign it to memory. Otherwise subsequent partitions will be applied on the original unpartitioned surface leading to the wrong outcome. Once the rectangular 
Published in: Finite Elements in Analysis and Design, 73C, 42-54.

DOI: http://dx.doi.org/10.1016/j.finel.2013.05.004

surface is fully partitioned, the nodes may be seeded and the mesh generated in the usual manner. The majority of the rectangular surface is thus meshed with orthogonal quadrilateral shell elements having the appropriate angle of inclination to the horizontal, while minor irregular regions near the boundaries may be filled in with triangular elements with the same order of shape function. The adverse effect of these irregular regions naturally diminishes as the mesh resolution is increased. A reasonable spacing between adjacent datum points is sufficient to ensure a correct mesh orientation, even for finer meshes. It is not necessary to generate very fine partitioning in order to obtain a fine mesh.

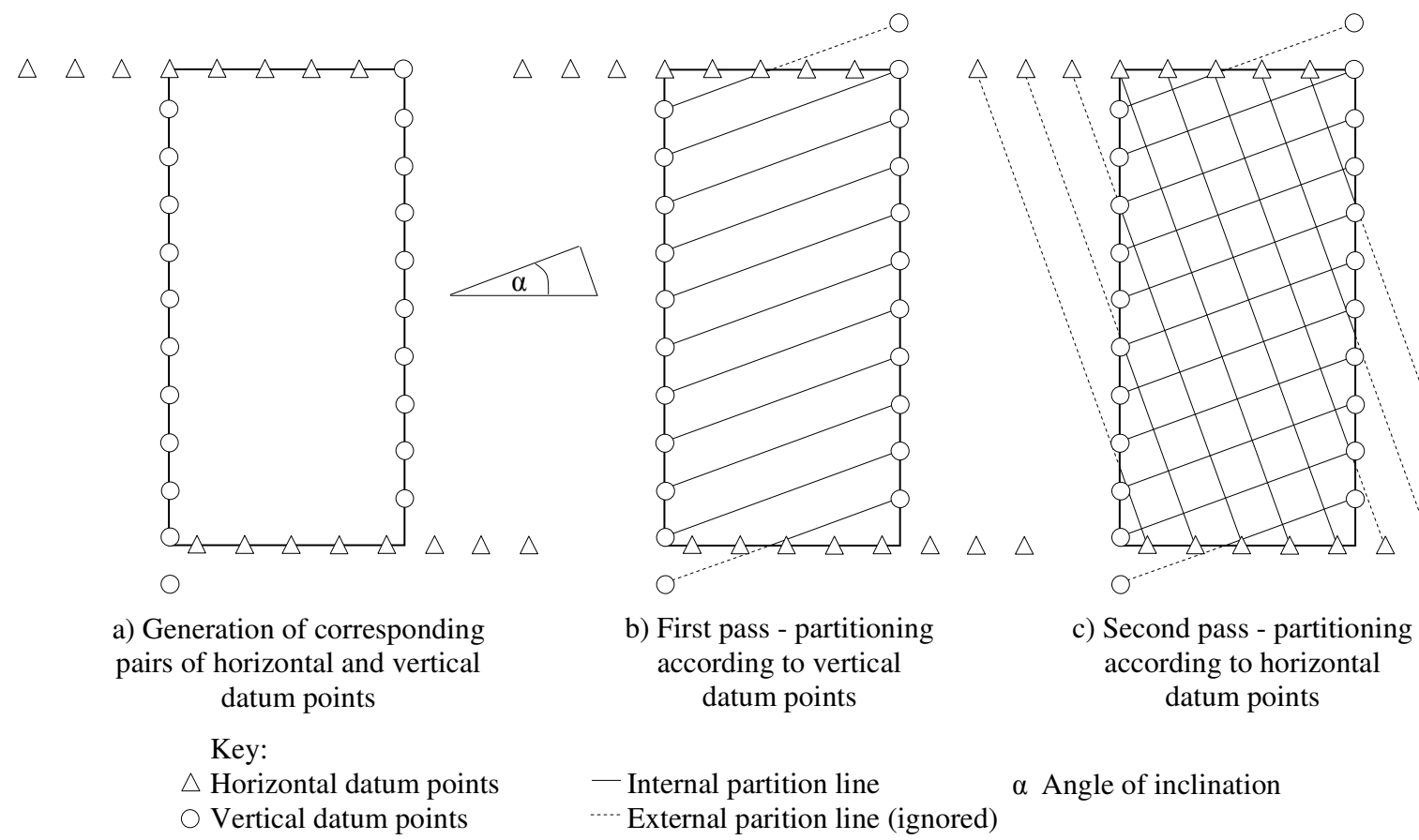

Fig. 2 - Progressive partitioning of a 2D plate into a well-structured inclined

orthogonal grid

\subsection{Thin 3D cylinder}

The method of meshing a 3D cylinder with inclined regular quadrilateral shell elements is significantly more complex due to the additional dimension. If to imagine the 3D cylinder as a rolled-up 2D rectangular strip, a mesh could be generated on the $2 \mathrm{D}$ rectangle in a manner similar to Fig. 2 and then mapped onto the $3 \mathrm{D}$ cylinder by an appropriate transformation, a method known as parametric meshing [28]. Though this would be the most efficient technique, commercial pre-processors do not support parametric meshing (i.e. a surface can only be meshed directly). It is thus again 
Published in: Finite Elements in Analysis and Design, 73C, 42-54.

DOI: http://dx.doi.org/10.1016/j.finel.2013.05.004

necessary to employ creative partitioning to force the meshing algorithm to conform to the axes of an inclined curvilinear grid, rather than of the original cylinder. This is achieved by partitioning the 'face' of the cylinder according to a family of adjacent orthogonal helices. Scripting is essential here.

A helix $H$ of radius $r$ may be defined by specifying either its angle of inclination to the horizontal axis $\alpha$ or the number of turns $n_{p}$ (not necessarily integer) per length $L$. These are related by:

$L=2 \pi r n_{p} \tan \alpha$

Let a helix $H_{1 m}$ be generated parametrically as follows $\left(\theta_{1} \in\left[0,2 \pi n_{p 1}\right]\right)$ :

$\left\{\begin{array}{l}x_{1}\left(\theta_{1}\right) \\ y_{1}\left(\theta_{1}\right) \\ z_{1}\left(\theta_{1}\right)\end{array}\right\}=\left\{\begin{array}{c}r \cos \left(\theta_{1}+\theta_{m}\right) \\ r \sin \left(\theta_{1}+\theta_{m}\right) \\ \left(P_{1} / 2 \pi\right) \theta_{1}\end{array}\right\}$ where $P_{1}=\frac{L}{n_{p 1}}=2 \pi r \tan \alpha$

A second helix $H_{2 m}$, orthogonal to the one above, may then be generated by $\left(\theta_{2} \in\left[0,2 \pi n_{p 2}\right]\right):$

$\left\{\begin{array}{l}x_{2}\left(\theta_{2}\right) \\ y_{2}\left(\theta_{2}\right) \\ z_{2}\left(\theta_{2}\right)\end{array}\right\}=\left\{\begin{array}{c}-r \cos \left(\theta_{2}+\theta_{m}\right) \\ r \sin \left(\theta_{2}+\theta_{m}\right) \\ \left(P_{2} / 2 \pi\right) \theta_{2}\end{array}\right\}$ where $P_{2}=\frac{L}{n_{p 2}}=2 \pi r \tan \left(\alpha+\frac{\pi}{2}\right)$

In the above, $P_{1}$ and $P_{2}$ represent the pitch of helices $H_{1 m}$ and $H_{2 m}$ respectively which is the change in the global $z$ coordinate per $2 \pi$ radians of the circumferential parameter $\theta_{1}$ or $\theta_{2}$. The subscript $m$ indicates that this is the $m$-th such pair of orthogonal helices, each pair being positioned at a circumferential offset $\theta_{m}$ given by:

$\theta_{m}=m \frac{2 \pi}{M}$ where $m \in[0, M-1]$ for a total of $M$ pairs of orthogonal helices

The partitioning procedure begins by generating datum points at the coordinates of the first $H_{1 m}$ helix (Eq. 2) with $m=0$ (Eq. 4). The cylinder is partitioned incrementally and in 'real time' according to the helical curve that is gradually generated on the cylinder 'face' between two immediately adjacent datum points along the same helix. The same operation is performed on an $M$ number of $H_{1}$-type helices, each separated by a circumferential offset of $2 \pi / M$ (Eq. 4). The procedure is then repeated on a further $M$ number of orthogonal $H_{2}$-type helices (Eq. 3). The resulting partition is illustrated in 
Published in: Finite Elements in Analysis and Design, 73C, 42-54.

DOI: http://dx.doi.org/10.1016/j.finel.2013.05.004

Fig. 3 for an inclination angle $\alpha$ of $45^{\circ}$. Once the cylinder surface is fully-partitioned, the mesh may be generated in the usual manner. Irregular regions near the top and bottom boundaries of the cylinder may be filled in with triangular elements of the same order. This procedure may become computationally expensive with very fine mesh resolutions.
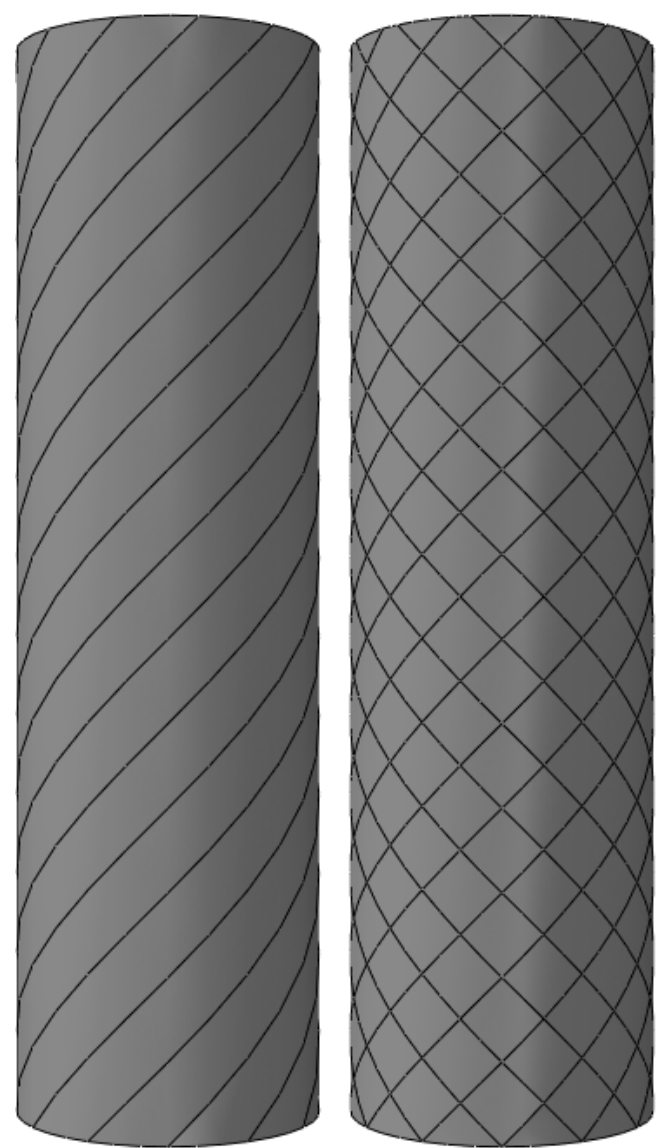

$1^{\text {st }}$ partitioning: $2^{\text {nd }}$ partitioning: $H_{1}$-type helices $H_{2}$-type helices

Fig. 3 - Progressive partitioning of a 3D cylinder according to orthogonal helices

\section{Benchmark Test \#1: linear buckling analysis of a thin 2D plate under uniform axial compression}

The first benchmark test to be considered was the linear-elastic buckling analysis of a thin plate subject to uniform in-plane compression along one pair of opposite edges, with pinned boundary conditions along all edges to prevent out-of-plane displacements. The classical thin-plate buckling solution [32,33] for this load case gives the critical shell edge load $N_{c l}$ as: 
Published in: Finite Elements in Analysis and Design, 73C, 42-54.

DOI: http://dx.doi.org/10.1016/j.finel.2013.05.004

$N_{c l}=k \frac{\pi^{2} D}{b^{2}}$ where $D=\frac{E t^{3}}{12\left(1-v^{2}\right)}$ and $k=\left(\frac{m b}{a}+\frac{a}{m b}\right)^{2}$
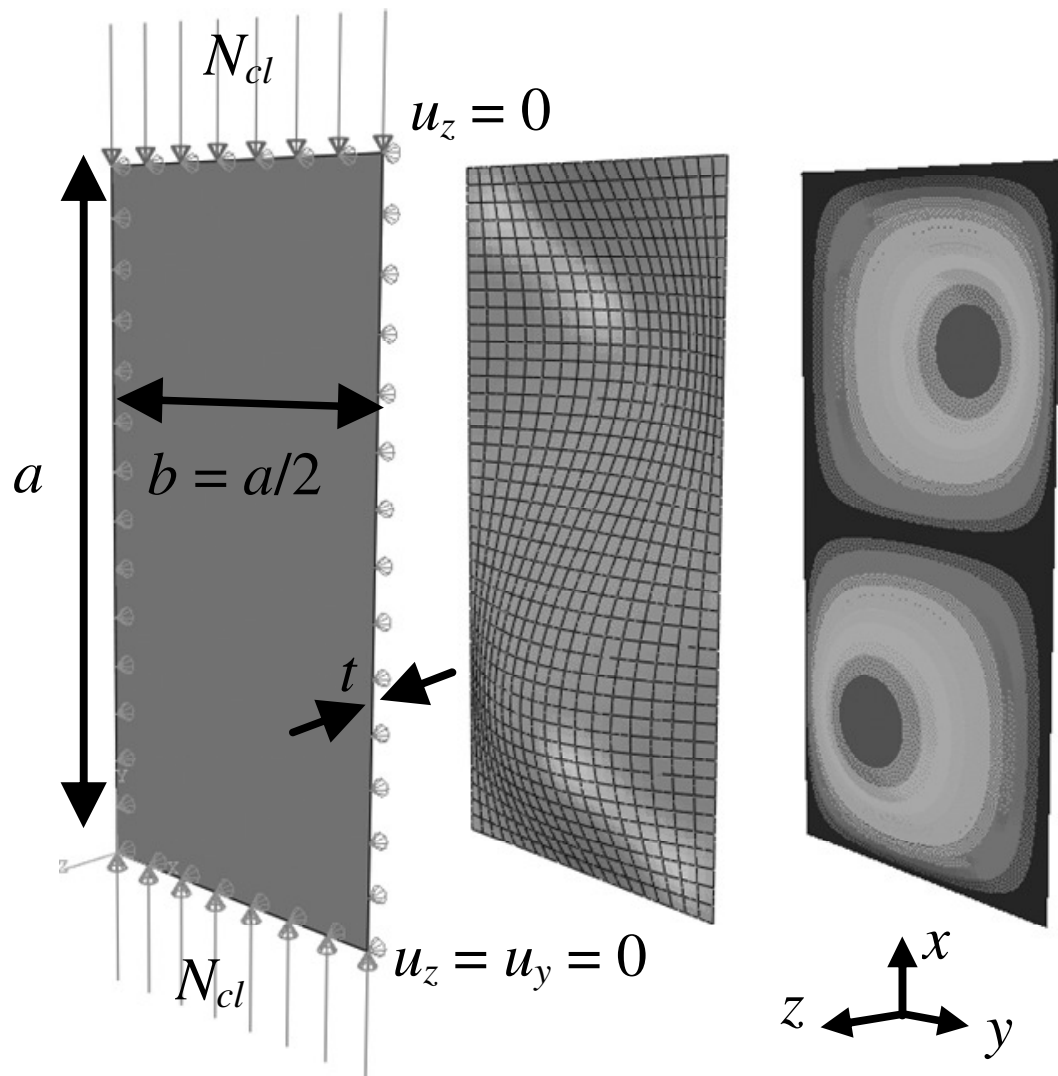

Fig. 4 - System geometry and critical bifurcation mode for the plate under uniform axial compression

In Eq. 5 above, $m$ is the wavelength parameter which should be chosen to minimise $k$. This is usually achieved by taking $m$ as the aspect ratio $a / b$ rounded down to the nearest integer. For an aspect ratio of 2, the correct linear bifurcation mode is a single sine half-wave in the direction transverse to the applied load $(x)$ and a full sine wave in the direction parallel to the load $(y)$. The parameters $E, v$ and $t$ represent the elastic modulus, Poisson's ratio and the plate thickness respectively. The numerical model, boundary conditions and critical buckling mode are illustrated in Fig. 4. The analyses were performed using ABAQUS v. 6.10.1 [31].

Quadrilateral-dominated meshes of increasing resolution were generated assuming inclinations of $\alpha=0,15,30$ and 45 degrees to the horizontal using the partitioning procedure described previously. It may be seen in Fig. 5 that the ABAQUS CAE 
Published in: Finite Elements in Analysis and Design, 73C, 42-54.

DOI: http://dx.doi.org/10.1016/j.finel.2013.05.004

meshing algorithm has trouble generating 'clean' inclined meshes for coarse element sizes, a problem that diminishes as the mesh resolution is increased. The most regular meshes were obtained for $\alpha=0^{\circ}$ and $45^{\circ}$. The meshes were chosen to consist of the ABAQUS S4R general-purpose 4-node reduced-integration shell elements, with triangular S3R elements as filler for the irregular boundary regions. The shear-flexible S4R element is widely used in computational studies within the shell buckling research community. Each mesh thus consisted of elements with a consistent order of shape function. An elastic modulus of $E=200 \mathrm{GPa}$ and a Poisson's ratio of $v=0.3$ were assumed, while the width to thickness ratio $b / t$ was taken as 100 . The results of the mesh convergence study are presented in Fig. 6 as the plot of the percentage difference between the numerical solution $N_{F E}$ and the classical solution $N_{c l}$ (Eq. 5) against the total number of the degrees of freedom (dofs) in the model. This information is presented in an alternative manner in Fig. 7 with the inverse of the number of dofs on the horizontal axis, possibly a clearer way of showing a variation with a dependent variable that tends to infinity. A number of observations may now be made.

Firstly, the numerical solution converges to a limiting value that is approximately $0.7 \%$ below than the classical result. This difference may be attributed to the fact that the formulation of a shear-flexible shell finite element is more complete with respect to the kinematic relations than what is assumed in the analytical treatment which is based on a Kirchhoff thin-plate approximation [30]. The difference is very small because of the particularly simple pre-buckling stress state governed solely by membrane equilibrium with the applied load and involving no plate bending (where the kinematic relations would play a more important role). Further, the plate assumed in these analyses has a $b / t$ ratio of 100 , which is not very thin, thus a discrepancy of this order of magnitude is to be expected. Indeed, a set of additional analyses on a plate with $b / t=1000$ revealed a much smaller limiting difference of approximately $0.05 \%$ and a similar outcome in terms of the effects of mesh orientation. 
Published in: Finite Elements in Analysis and Design, 73C, 42-54.

DOI: http://dx.doi.org/10.1016/j.finel.2013.05.004

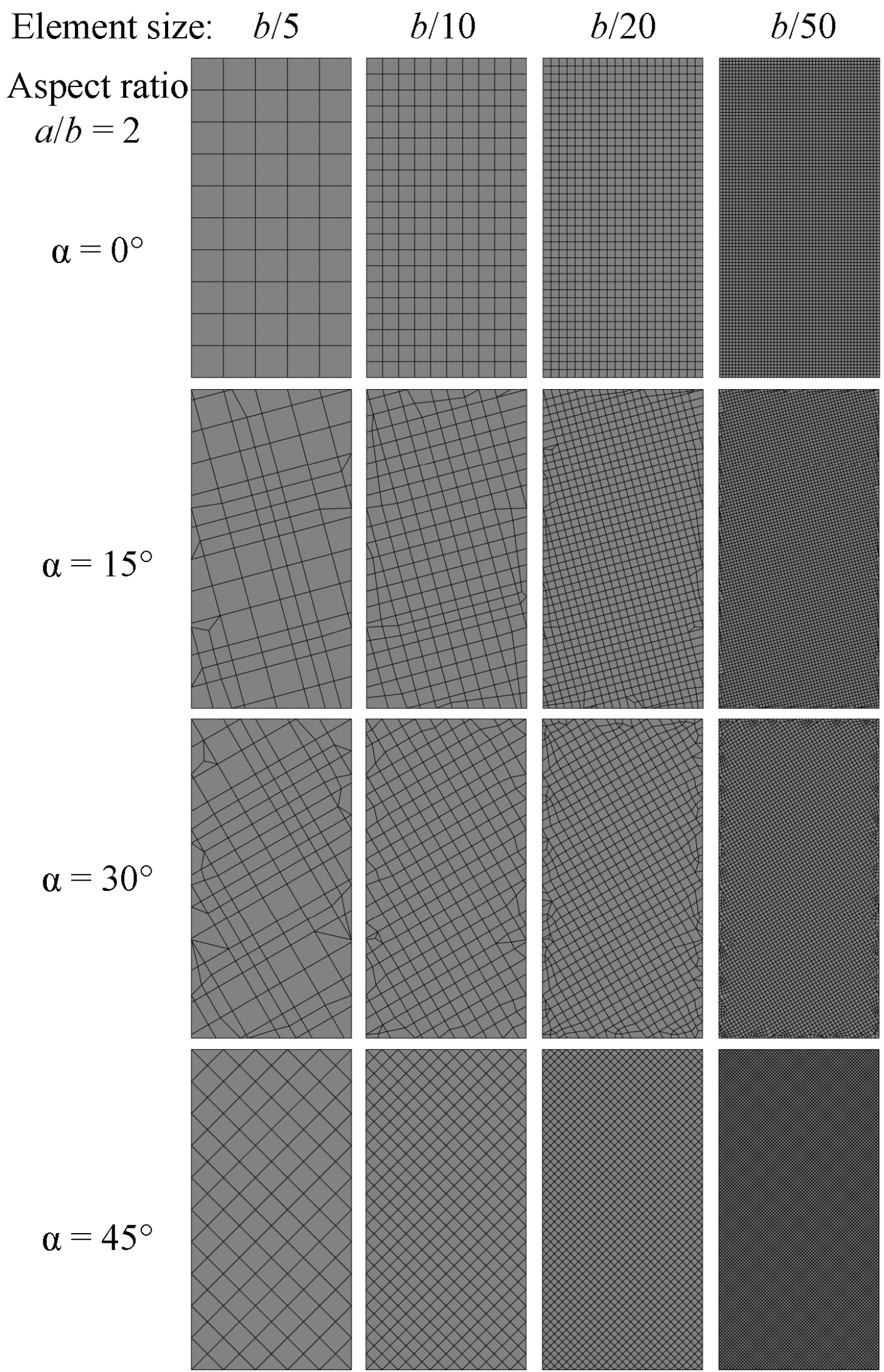

Fig. 5 - Increasingly fine inclined finite element meshes used for the analysis of the plate 
Published in: Finite Elements in Analysis and Design, 73C, 42-54.

DOI: http://dx.doi.org/10.1016/j.finel.2013.05.004

Secondly, the convergence occurs 'from above' as a consequence of the Rayleigh-Ritz principle [34]. As stated above, the pre-buckling stress state in this structure is completely dominated by membrane equilibrium with the applied axial compressive load. The destabilising stresses are thus evaluated 'correctly'. However, the displacement field of the finite element model is a piecewise linear approximation to the 'real' solution, an over-constraint which inevitably leads to an excessive stiffness in the system. In order to compensate for this additional stiffness, the critical buckling load will be higher than it should be and will therefore converge to the correct value 'from above' as the mesh resolution increases.

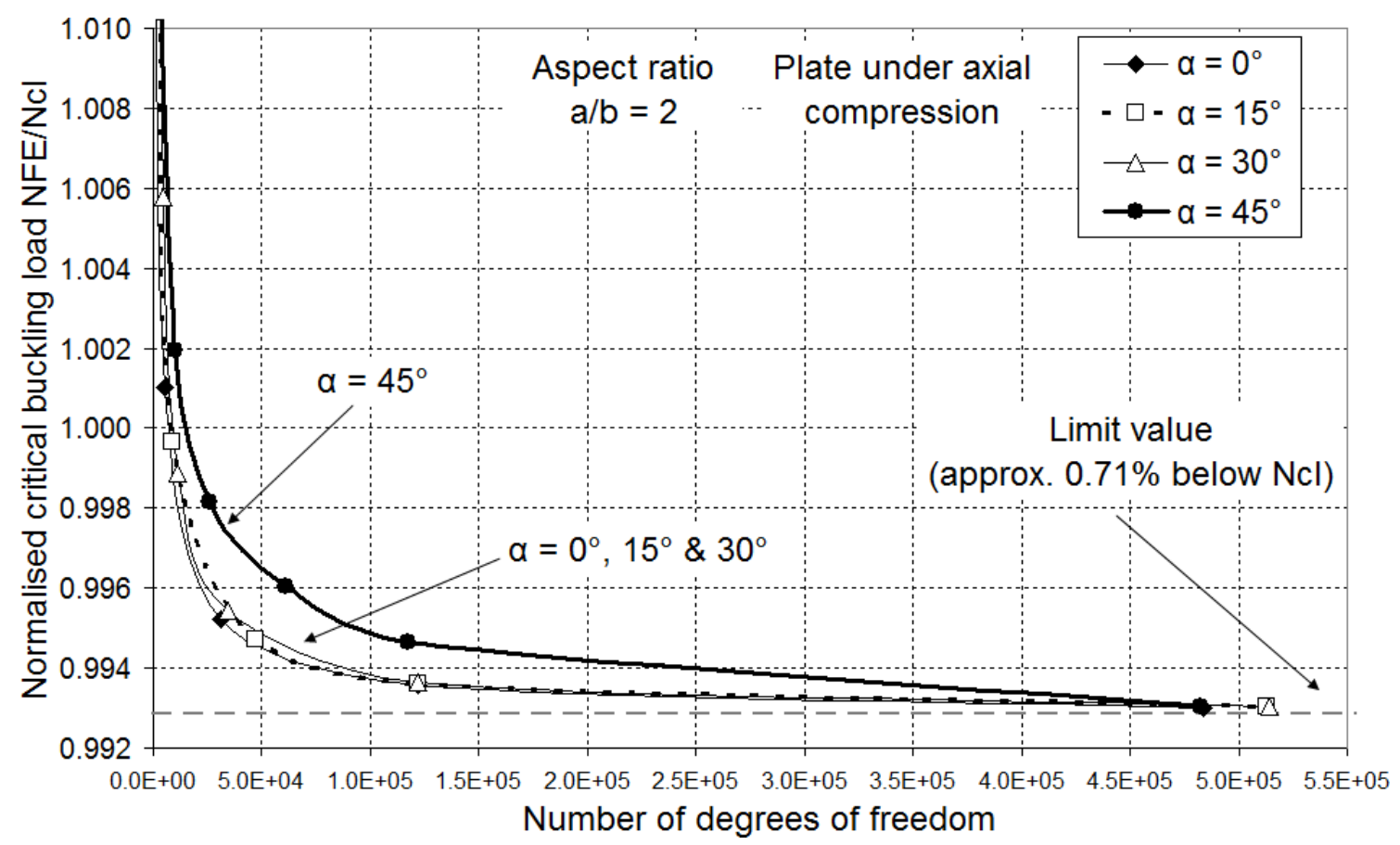

Fig. 6 - Mesh convergence with total dofs for the plate under axial compression 
Published in: Finite Elements in Analysis and Design, 73C, 42-54.

DOI: http://dx.doi.org/10.1016/j.finel.2013.05.004

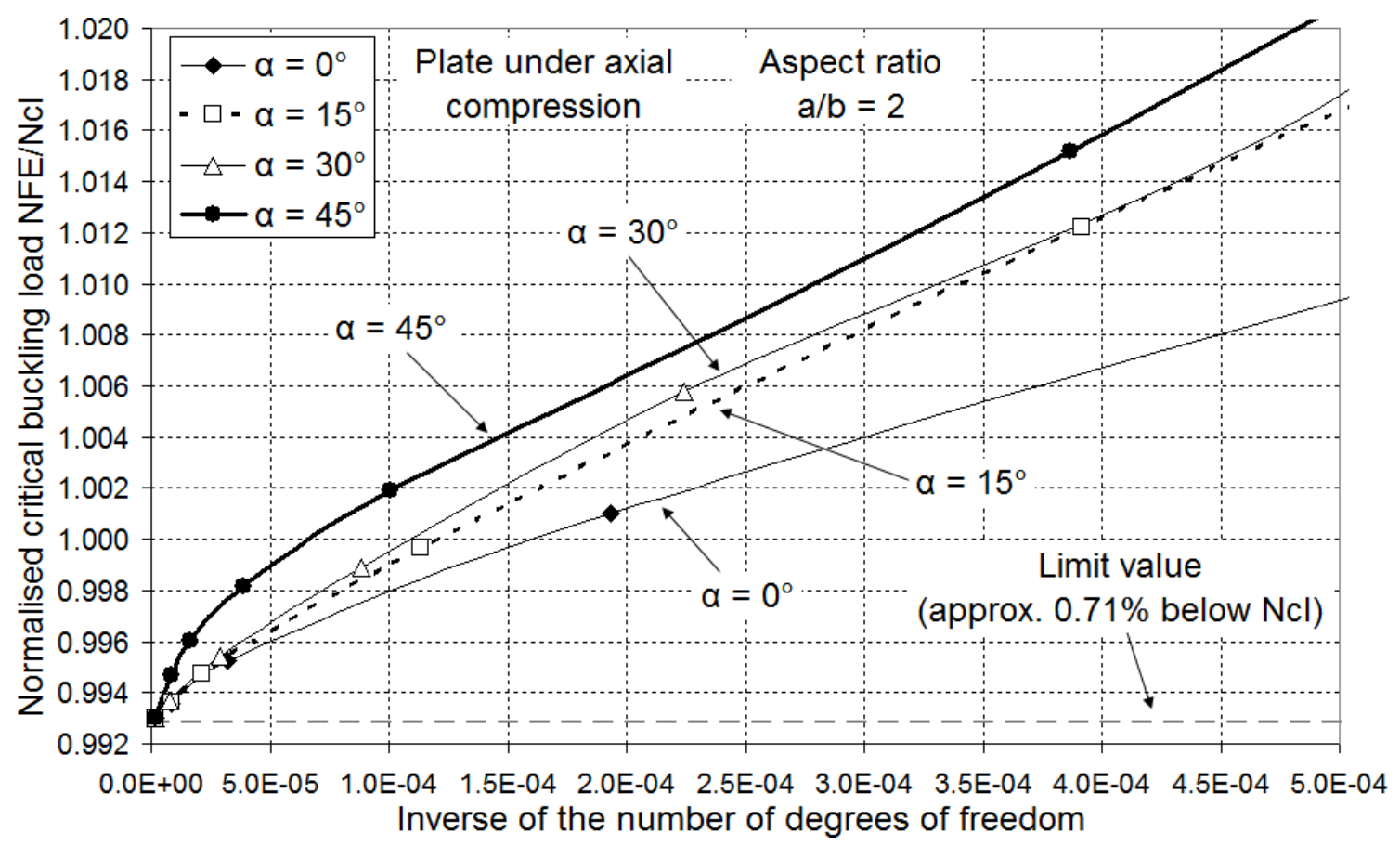

Fig. 7 - Mesh convergence with inverse of dofs for the plate under axial compression

Lastly, meshes inclined at $15^{\circ}$ and $30^{\circ}$ performed only marginally worse than the regular rectangular mesh $\left(\alpha=0^{\circ}\right)$, suggesting that the accuracy of the solution or the rate of convergence is not very sensitive to the mesh orientation. The exception to this proved to be the mesh inclined at $45^{\circ}$, which performed particularly poorly. For example, $87 \%$ and $103 \%$ more dofs were required to achieve $2 \%$ and $1 \%$ errors respectively with the limit value for $\alpha=45^{\circ}$ than for the regular rectangular mesh. The reason for this is that elements with $\alpha=45^{\circ}$ are subject to pure shear when the plate is under uniform axial compression. These results therefore support the hypothesis that the orientation of the elements with respect to the principal axes of stress may be an important consideration in the finite element modelling of problems in structural mechanics, since there may exist a particularly unfortunate orientation at which the convergence of the inclined mesh will be at its worst.

\section{Benchmark Test \#2: linear buckling analysis of a thin 2D plate under uniform shear}

The second benchmark test to be performed was the linear-elastic buckling analysis of a rectangular plate under uniform edge shear. The classical solution gives [32,33] the critical value of edge shear $N_{x y, c l}$ as: 
Published in: Finite Elements in Analysis and Design, 73C, 42-54.

DOI: http://dx.doi.org/10.1016/j.finel.2013.05.004

$N_{x y, c l}=k \frac{\pi^{2} D}{b^{2}}$ where $D=\frac{E t^{3}}{12\left(1-v^{2}\right)}$ and $k \approx 6.6$ for an aspect ratio $a / b$ of 2

The above value of $k$ is based on an approximate solution to a complex minimisation problem and is known to have an error of the order of $1 \%$ (further details may be found in the above reference). The geometry, boundary conditions and critical linear buckling mode for this problem are illustrated in Fig. 8. The same types of meshes were employed using ABAQUS as for the first benchmark test (Fig. 5) with the same assumed angles of inclination.
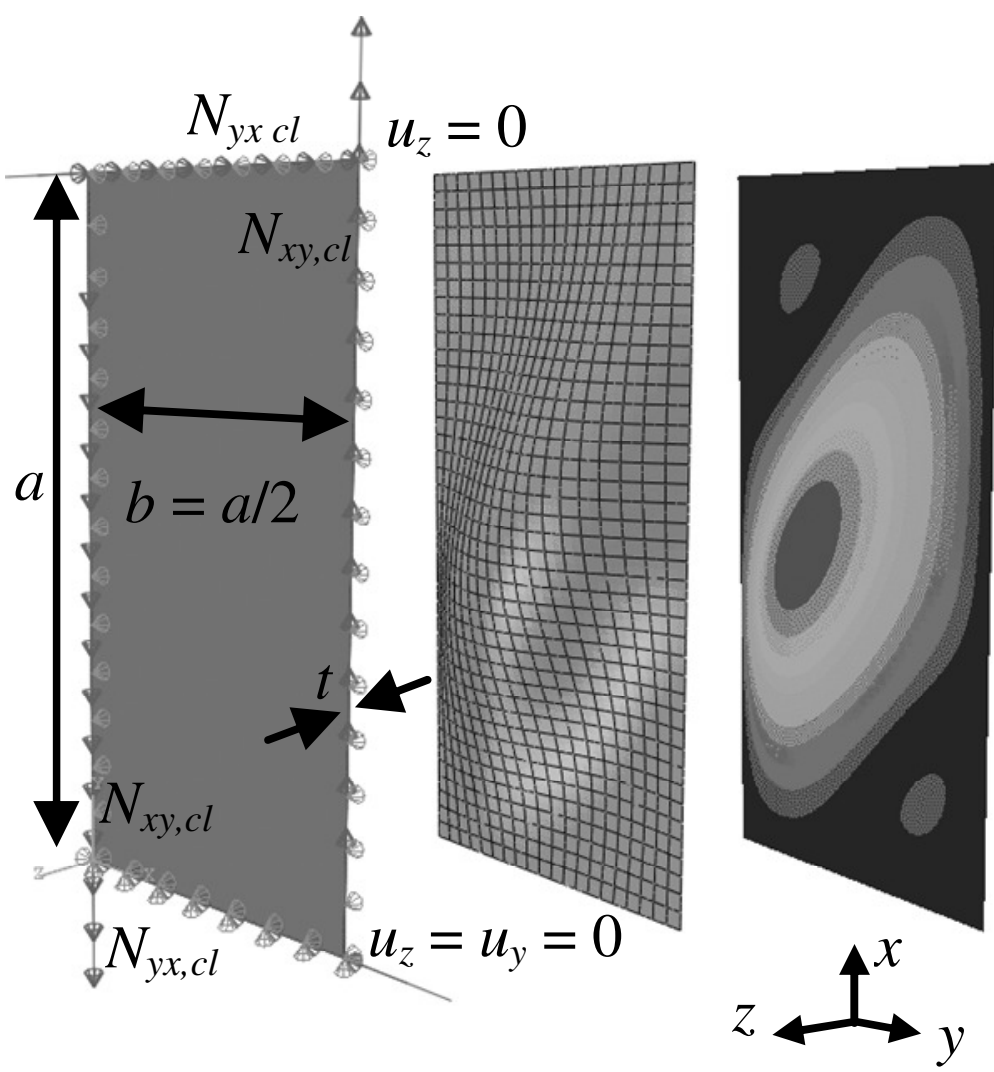

Fig. 8 - System geometry and critical bifurcation mode for the plate under uniform edge shear

The results of the mesh convergence analyses for the plate under uniform edge shear are presented in Figs 9 and 10 in a similar manner to the plate under axial compression. The convergence of $N_{F E} / N_{x y, c l}$ occurs 'from above' for the plate under shear, for similar reasons as for the plate under axial compression. The analyses for the plate with $b / t=$ 100 converge to a limiting value that is approximately $1.2 \%$ lower than Eq. 6, while a complementary set of analyses performed on a much thinner plate (not shown) with $b / t$ 
Published in: Finite Elements in Analysis and Design, 73C, 42-54.

DOI: http://dx.doi.org/10.1016/j.finel.2013.05.004

$=1000$ converges to a limiting difference of approximately $0.8 \%$ while exhibiting a similar relationship with the mesh orientation. These values are consistent with the order of magnitude error that Eq. 6 is known to have [32]. The smaller limiting difference for $b / t=1000$ may be explained by the fact that such a thin plate corresponds more closely to the Kirchhoff thin-plate assumptions of the analytical solution (Eq. 6) than a plate with $b / t=100$.

In direct contrast to the plate under axial compression, the regular mesh $\left(\alpha=0^{\circ}\right)$ now exhibits the slowest rate of convergence because for this particular system the 'default' mesh orientation subjects the elements directly to pure shear and thus requires the finest meshes to achieve a desired accuracy. The best performance was found in almost equal measure for the meshes with $\alpha=30^{\circ}$ and $45^{\circ}$, whose inclined elements are closest to being orthogonal to the midline through the buckle which is $\operatorname{at}^{-1}(a / b)=$ $\tan ^{-1}(2) \approx 63^{\circ}$ to the horizontal. In particular, the regular mesh with $\alpha=0^{\circ}$ required $40 \%$ and $45 \%$ more elements than $\alpha=45^{\circ}$ to achieve a $2 \%$ and $1 \%$ error respectively to the limit value. Interestingly, even an inclination of $15^{\circ}$ already exhibits a noticeable improvement in performance over the regular mesh with $\alpha=0^{\circ}$, requiring only $14 \%$ and $20 \%$ more elements than $\alpha=45^{\circ}$ to achieve the same respective percentage errors as noted above. This is significant because many finite element analysts may use the regular rectangular mesh for this problem simply on the basis that the plate is also rectangular and without being aware that this most obvious 'default' mesh orientation is also the least suitable for this particular problem. 
Published in: Finite Elements in Analysis and Design, 73C, 42-54.

DOI: http://dx.doi.org/10.1016/j.finel.2013.05.004

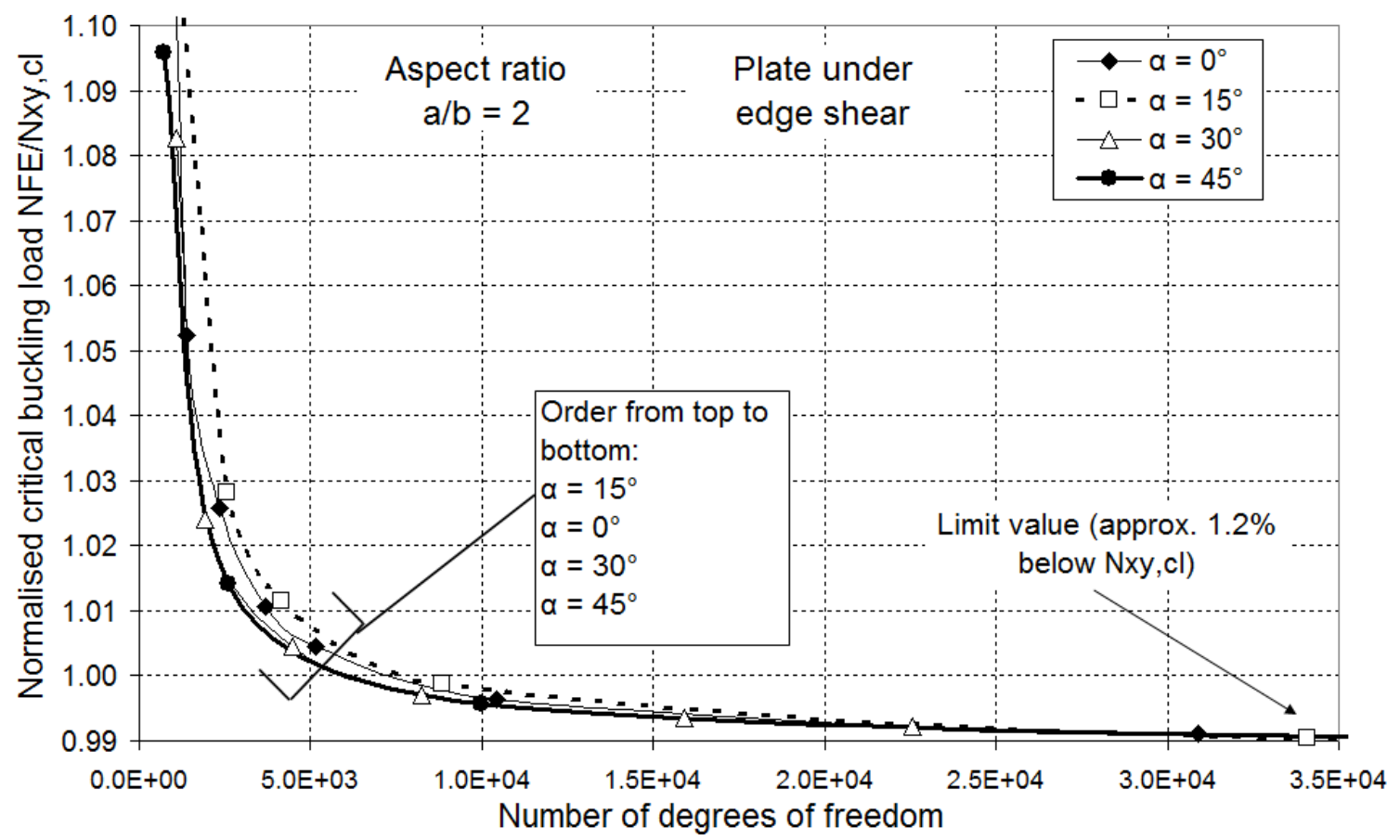

Fig. 9 - Mesh convergence with total dofs for the plate under edge shear

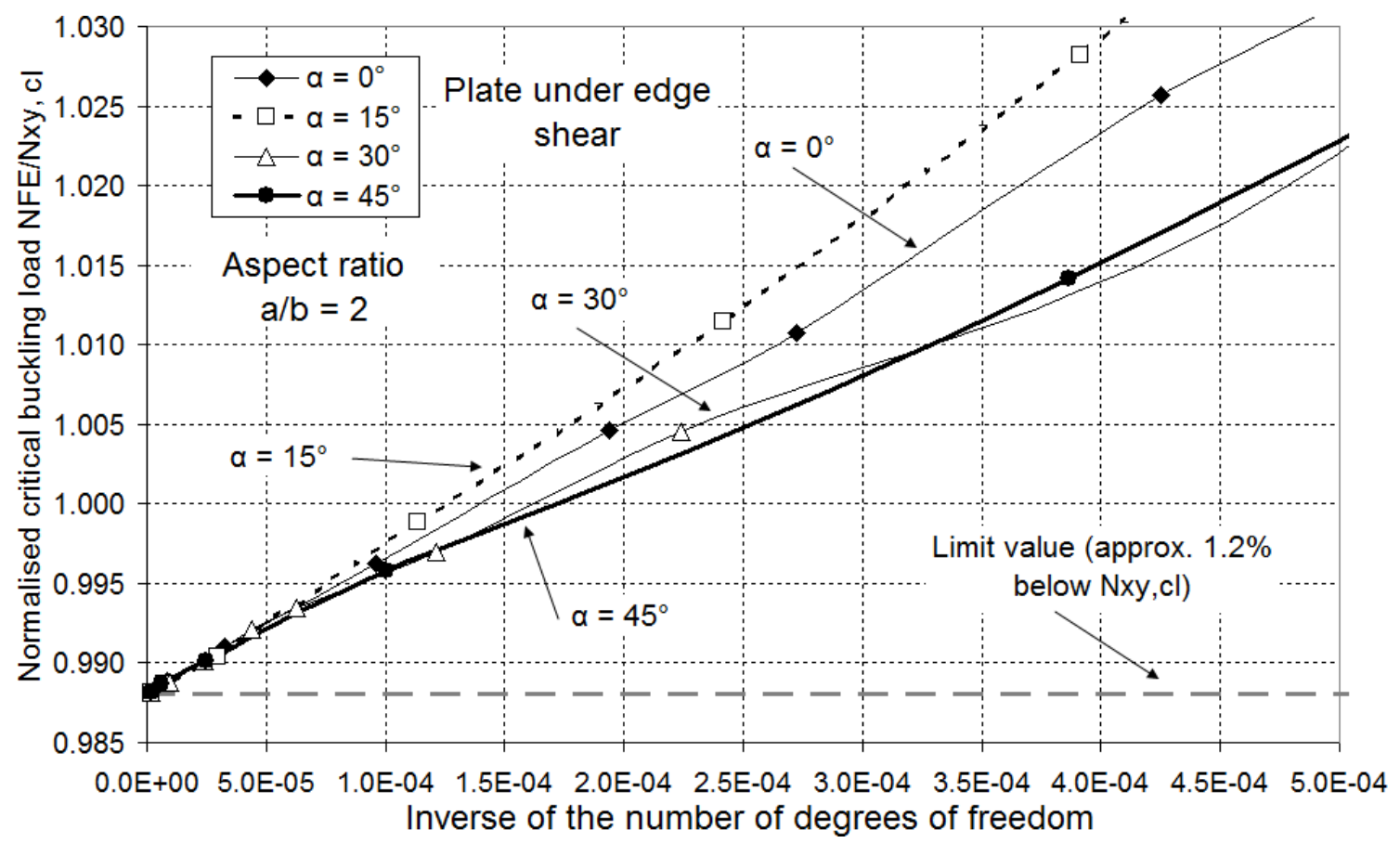

Fig. 10 - Mesh convergence with inverse of dofs for the plate under edge shear 
Published in: Finite Elements in Analysis and Design, 73C, 42-54.

DOI: http://dx.doi.org/10.1016/j.finel.2013.05.004

\section{Benchmark Test \#3: linear buckling analysis of a thin 3D cylinder under uniform axial compression}

The next benchmark test to be performed was the linear buckling analysis of a 3D axisymmetric cylinder under uniform axial compression. This is a ubiquitous standard case in the analysis of thin cylindrical shells and one with a well-understood, albeit complex, behaviour. The classical solution $[32,33]$ for the critical axial load $P_{c l}$, assuming pinned boundary conditions at either end, is given by:

$P_{c l}=2 \pi r t \sigma_{c l}$ where $\sigma_{c l}=\frac{E t}{r \sqrt{3\left(1-v^{2}\right)}}$

In the finite element model, the load $P_{c l}$ was applied through a reference point linked to the nodes of one end of the cylinder by a rigid body kinematic coupling, which also ensured that this end remained circular during the analysis. This reference point was then restrained against radial and circumferential displacement. The other end of the cylinder was left unloaded but restrained against all displacements. The shell was free to undergo axial rotation at either end. The cylinder was assumed to have an aspect ratio of $l / r=7$ (i.e. 'medium' length) and a radius to thickness ratio of $r / t=100$. The critical buckling mode is predicted analytically to be a global 'chequerboard' pattern with approximately $n_{c l} \approx 0.909 \sqrt{ }(r / t) \approx 9$ square waves around the circumference, though in fact very many modes are almost simultaneously critical for this load case [2,35]. An elastic modulus of $E=200 \mathrm{GPa}$ and a Poisson's ratio of $v=0.3$ were again assumed. First-order finite element meshes (ABAQUS shell elements S4R + S3R as filler) of increasing resolution were generated with angles of inclination of $0,15,30$ and 45 degrees to the horizontal according to the helical partitioning procedure described earlier. 
Published in: Finite Elements in Analysis and Design, 73C, 42-54.

DOI: http://dx.doi.org/10.1016/j.finel.2013.05.004

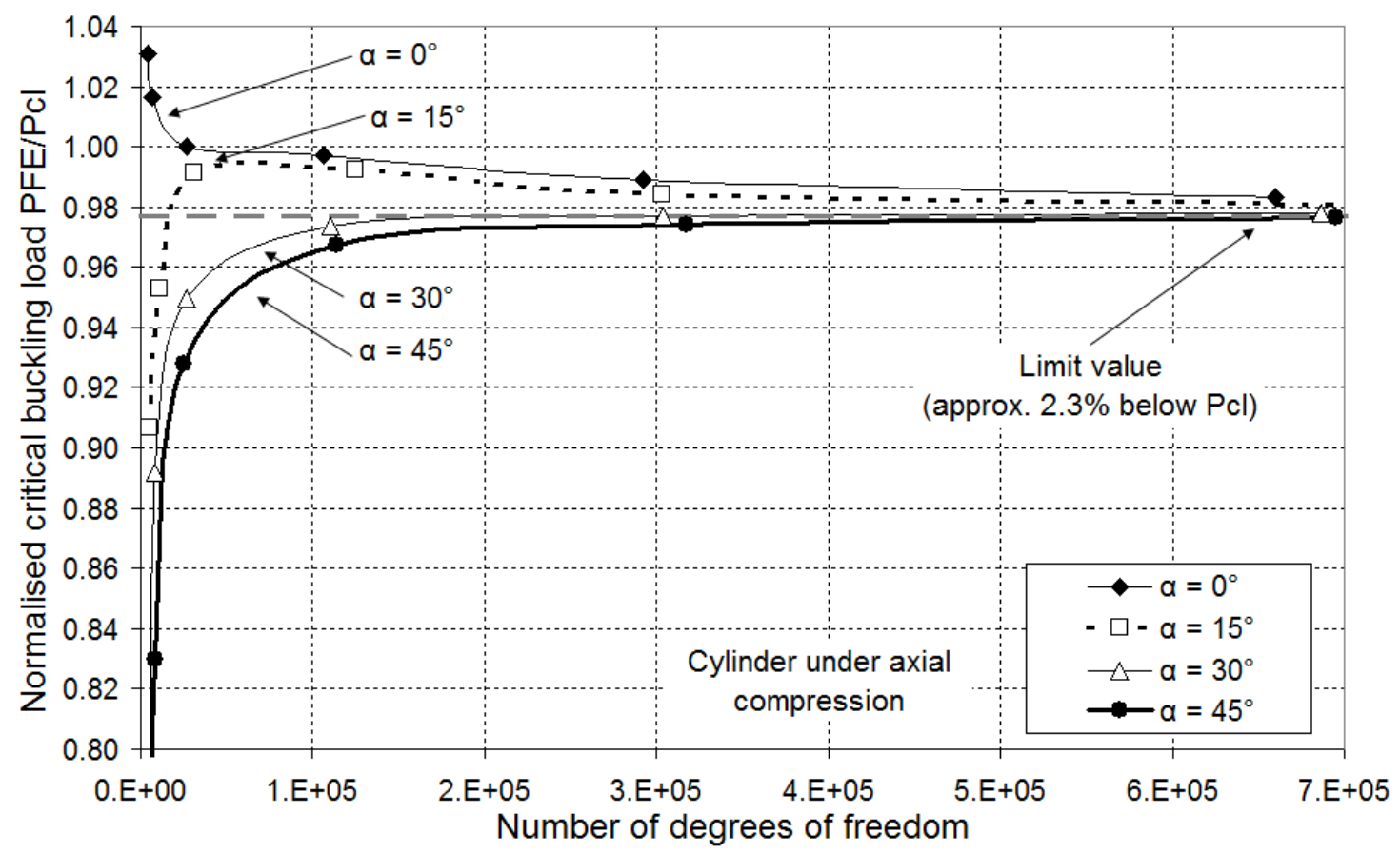

Fig. 11 - Mesh convergence with total dofs for the cylinder under uniform axial compression

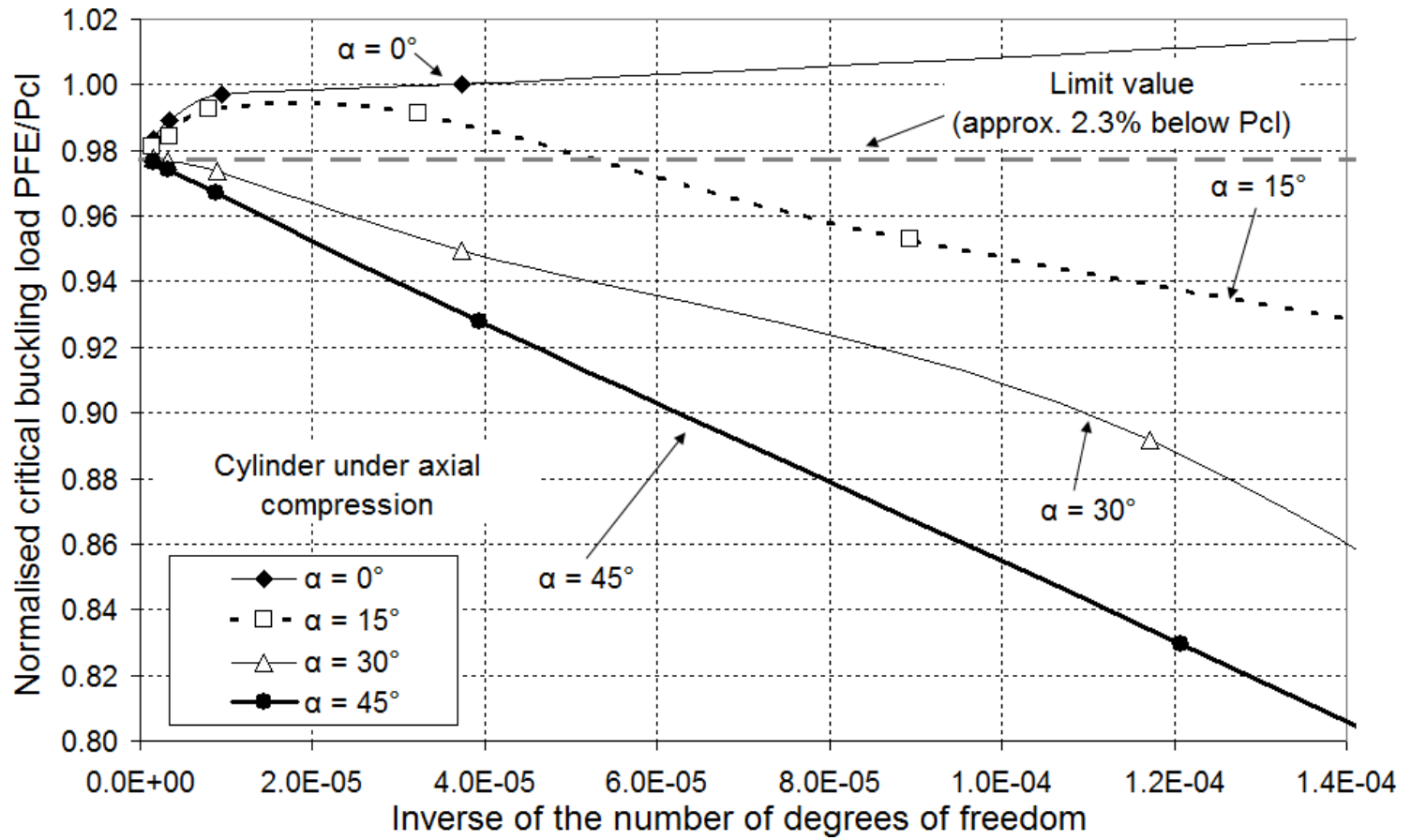

Fig. 12 - Mesh convergence with inverse of dofs for the cylinder under uniform axial compression

The convergence of $P_{F E} / P_{c l}$ is illustrated in Figs 11 and 12. The analyses converge to a critical value that is approximately $2.3 \%$ lower than the analytical solution $P_{c l}$ (Eq. 7). 
Published in: Finite Elements in Analysis and Design, 73C, 42-54.

DOI: http://dx.doi.org/10.1016/j.finel.2013.05.004

This difference may again be attributed to the fact that the analytical solution is based on the 'Donnell' form of equilibrium equations with simplified kinematic relations appropriate for 'thin' shells [36], whereas the formulation of the general-purpose S4R shell finite element includes more complete kinematic relations. The helical mesh inclined at $\alpha=45^{\circ}$ exhibits the slowest convergence and the least accurate prediction of the critical buckling load because the elements are subject to pure shear, consistent with the behaviour of the axially-compressed plate (Figs 6 and 7). However, the regular mesh with $\alpha=0^{\circ}$ achieves a superior accuracy with fewer elements only at first, and eventually the meshes with $\alpha=30^{\circ}$ and $45^{\circ}$ require $70 \%$ and $54 \%$ respectively fewer dofs than $\alpha=0^{\circ}$ to achieve a $1 \%$ level of error with the limit value. This suggests that the optimal mesh orientation for this deceptively simple load condition may actually be an inclined one. It is not immediately clear why this is so.

An even more curious finding, and one that is harder to explain, is why meshes with $\alpha$ $=0^{\circ}$ (consistently) and $15^{\circ}$ (only partially) converge 'from above' as expected based on the Rayleigh-Ritz principle [34], but meshes with $\alpha=30^{\circ}$ and $45^{\circ}$ appear to converge 'from below'. The reason for this may lie in the fact that the boundary conditions have a more complex influence on the pre-buckling stress state for a $3 \mathrm{D}$ cylinder than for a $2 \mathrm{D}$ plate. In particular, the cylindrical shell must undergo axial bending in order to maintain compatibility with the radial restraint at the bottom boundary, thus the prebuckling membrane stress state is no longer governed by membrane equilibrium alone. The stresses near the bottom boundary are therefore overestimated as they are dependent on a displacement field that is overly stiff due to its piecewise linear constraint, which in turn leads to an underestimated critical buckling load. This effect appears to dominate when the mesh is inclined, giving convergence 'from below', but not for a regular or a gently inclined mesh where convergence is instead 'from above'. It is not yet clear why the inclination of the mesh leads to such a fundamental change in the convergence behaviour of this system. 
Published in: Finite Elements in Analysis and Design, 73C, 42-54.

DOI: http://dx.doi.org/10.1016/j.finel.2013.05.004
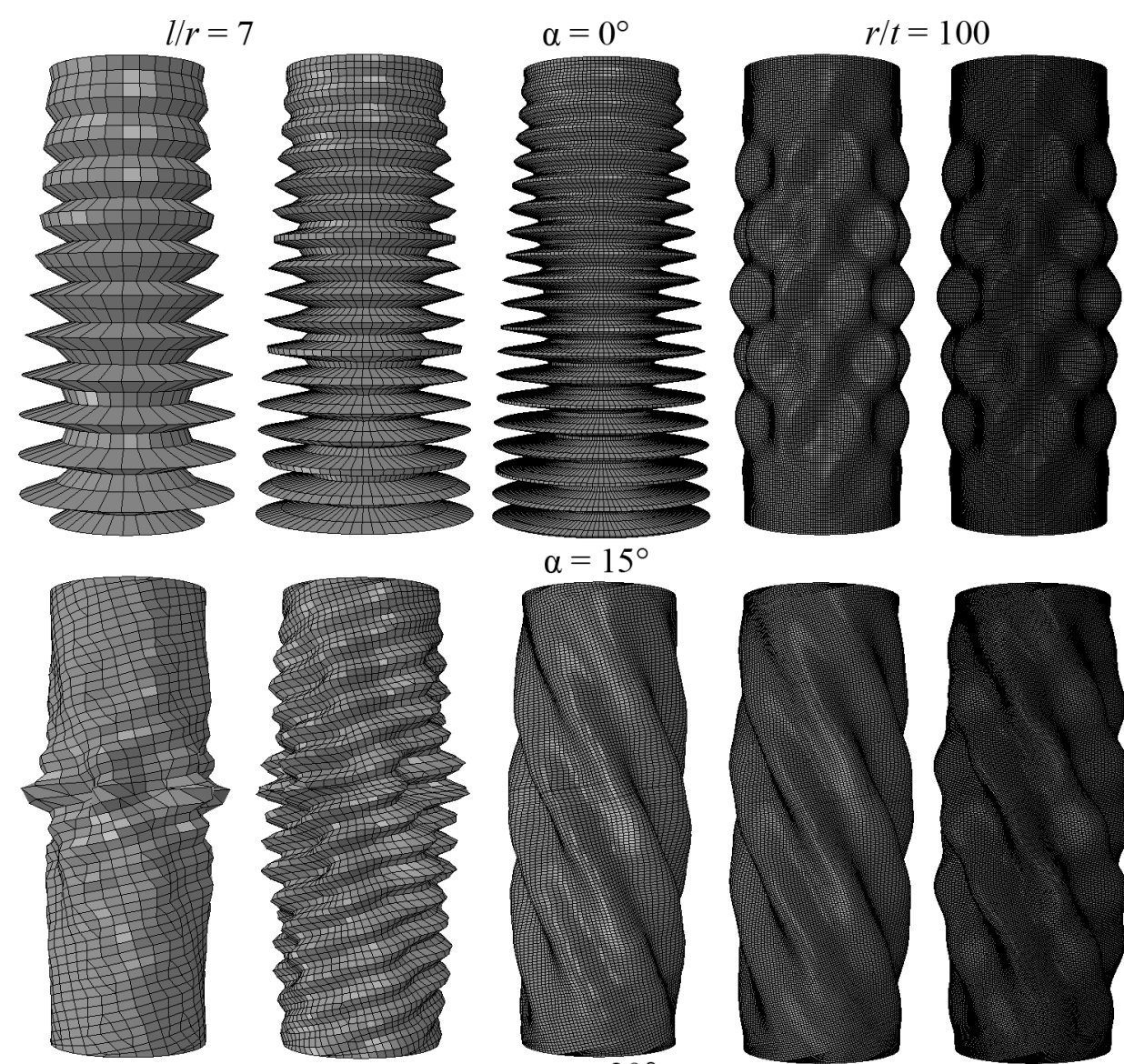

$$
\alpha=15^{\circ}
$$
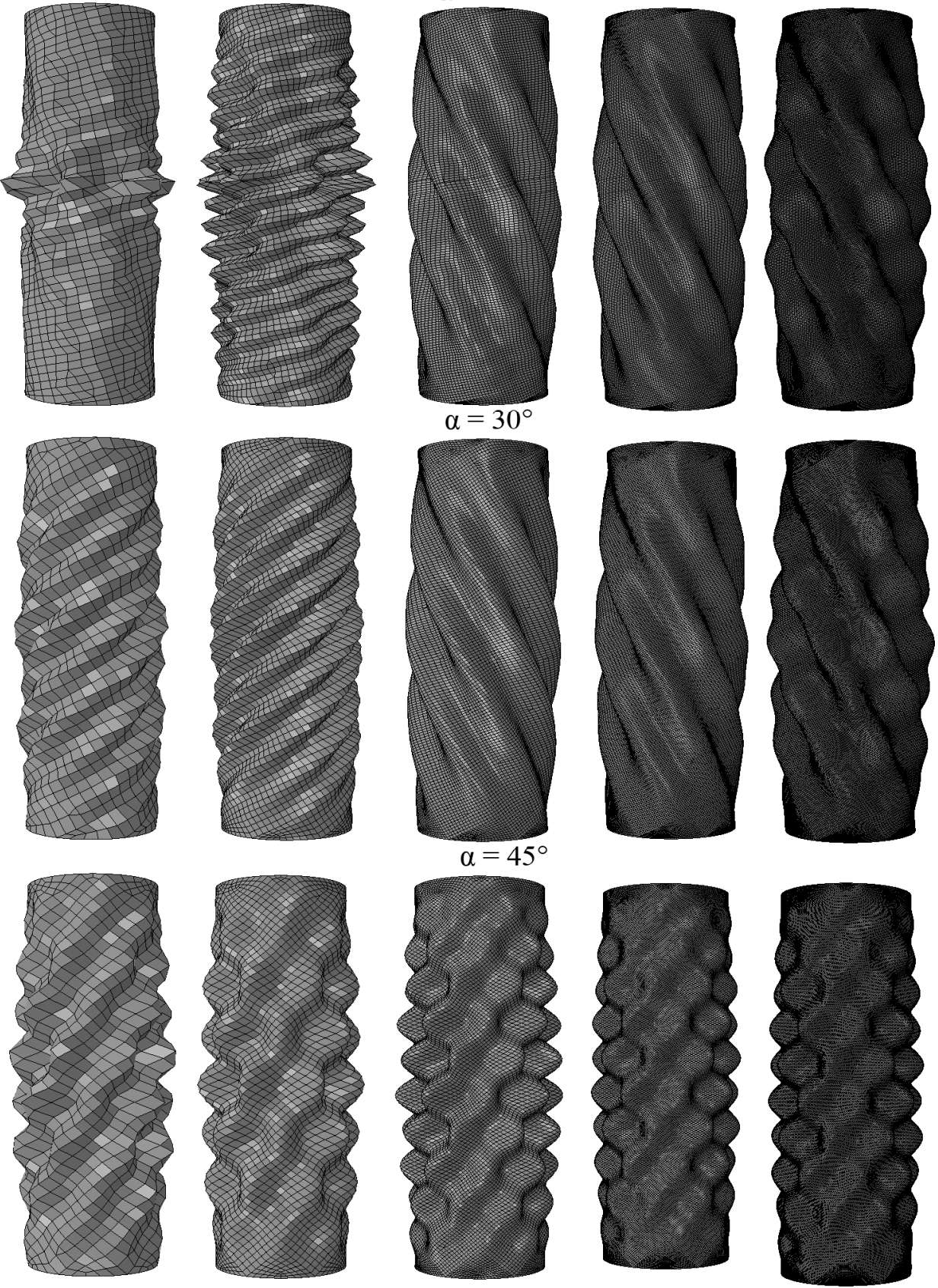

Fig. 13 - Computed linear bifurcation modes for the cylinder under axial compression 
Published in: Finite Elements in Analysis and Design, 73C, 42-54.

DOI: http://dx.doi.org/10.1016/j.finel.2013.05.004

The computed buckling modes are shown in Fig. 13 for the different mesh resolutions and inclinations. The modes for the three coarsest meshes with $\alpha=0^{\circ}$ are of the 'axisymmetric' type with a single circumferential wave and what appears to be an abrupt variation near the bottom end that may be attributed to the unrestrained axial rotation at this boundary condition and linear element shape functions. More importantly, though the critical buckling load clearly converges to the same value regardless of mesh orientation (correct to within $0.1 \%$ of the critical load for the regular mesh), the critical buckling modes are quite different. For the finest mesh resolutions, a mesh with $\alpha=0^{\circ}$ exhibits a 'chequerboard' pattern of square buckles with 7 circumferential waves, but a mesh with $\alpha=45^{\circ}$ predicts 8 waves instead. Meshes with $\alpha=15^{\circ}$ and $30^{\circ}$ exhibit interesting 'spiral' buckling modes that appear to follow the angle of the helical mesh. The reason for this is that the helical nature of the mesh acts as a very minor imperfection which, though too minor to influence the critical load, is significant enough to bias the buckling mode to lie in a more favourable orientation relative to the axis of the helix. The near-simultaneous occurrence of many distinctly different critical buckling modes very close to the same critical value is a manifestation of the particularly acute imperfection sensitivity of the axiallycompressed cylinder [35].

\section{Benchmark Test \#4: linear buckling analysis of a thin 3D cylinder under uniform end torque}

The final benchmark test to be considered was the linear buckling analysis of a cylinder subject to uniform torsion at one end. Timoshenko and Gere [32] offer the following approximate analytical formula for the critical torque moment $T_{c l}$ for medium-length shells based on the simplified Donnell kinematic relations:

$T_{c l}=2 \pi r^{2} t \tau_{c l}$ where $\tau_{c l}=4.39 \frac{E t^{2}}{l^{2}\left(1-v^{2}\right)} \sqrt{1+0.0257\left(1-v^{2}\right)^{0.75}\left(\frac{l}{\sqrt{r t}}\right)^{3}}$

The finite element model was constructed in the same manner as for the cylinder under axial compression, except that a torque $T_{c l}$ (moment about the vertical axis) was applied through the end reference point in place of the axial load. The same model geometry and first-order element mesh orientations were also assumed. 
Published in: Finite Elements in Analysis and Design, 73C, 42-54.

DOI: http://dx.doi.org/10.1016/j.finel.2013.05.004

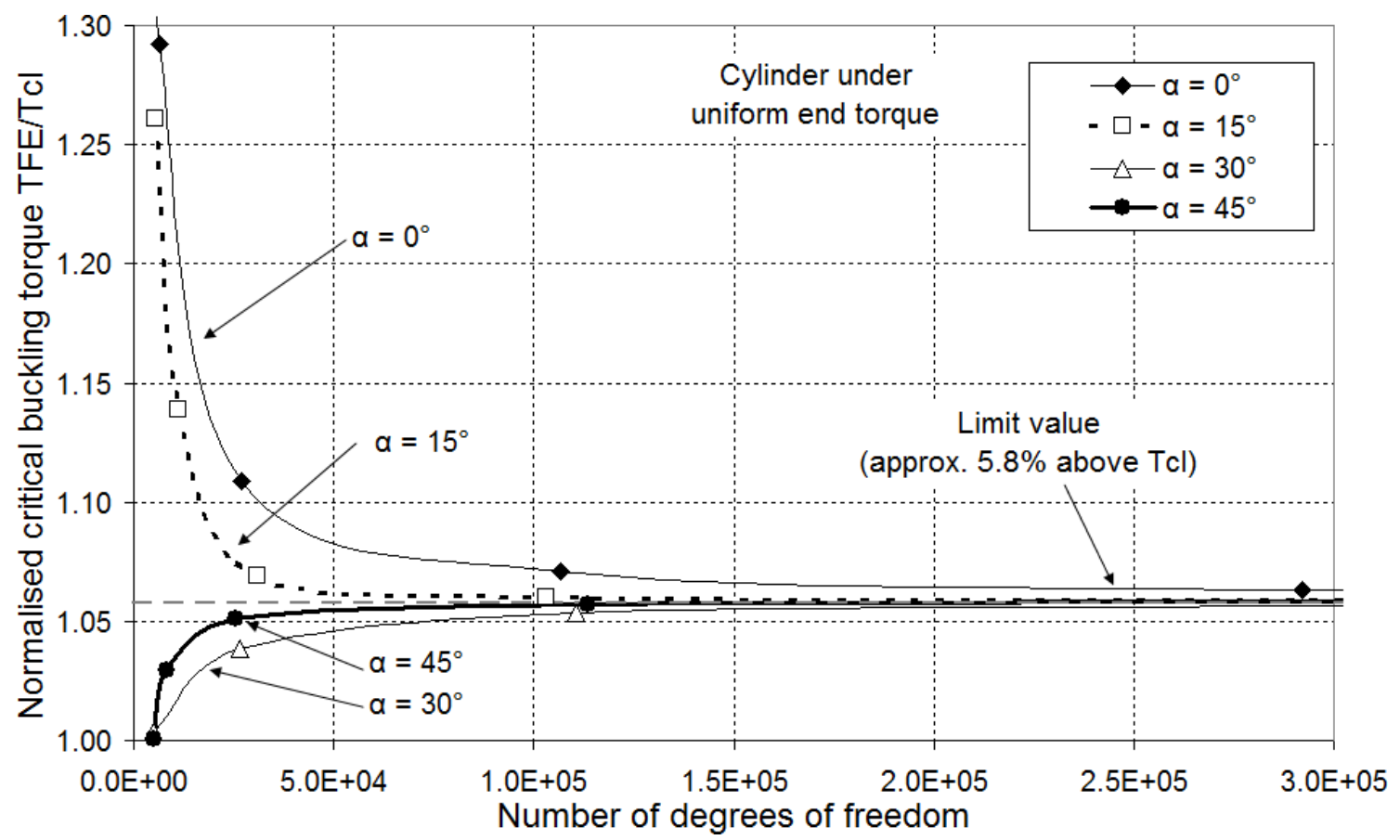

Fig. 14 - Mesh convergence with total dofs for the cylinder under uniform end torque

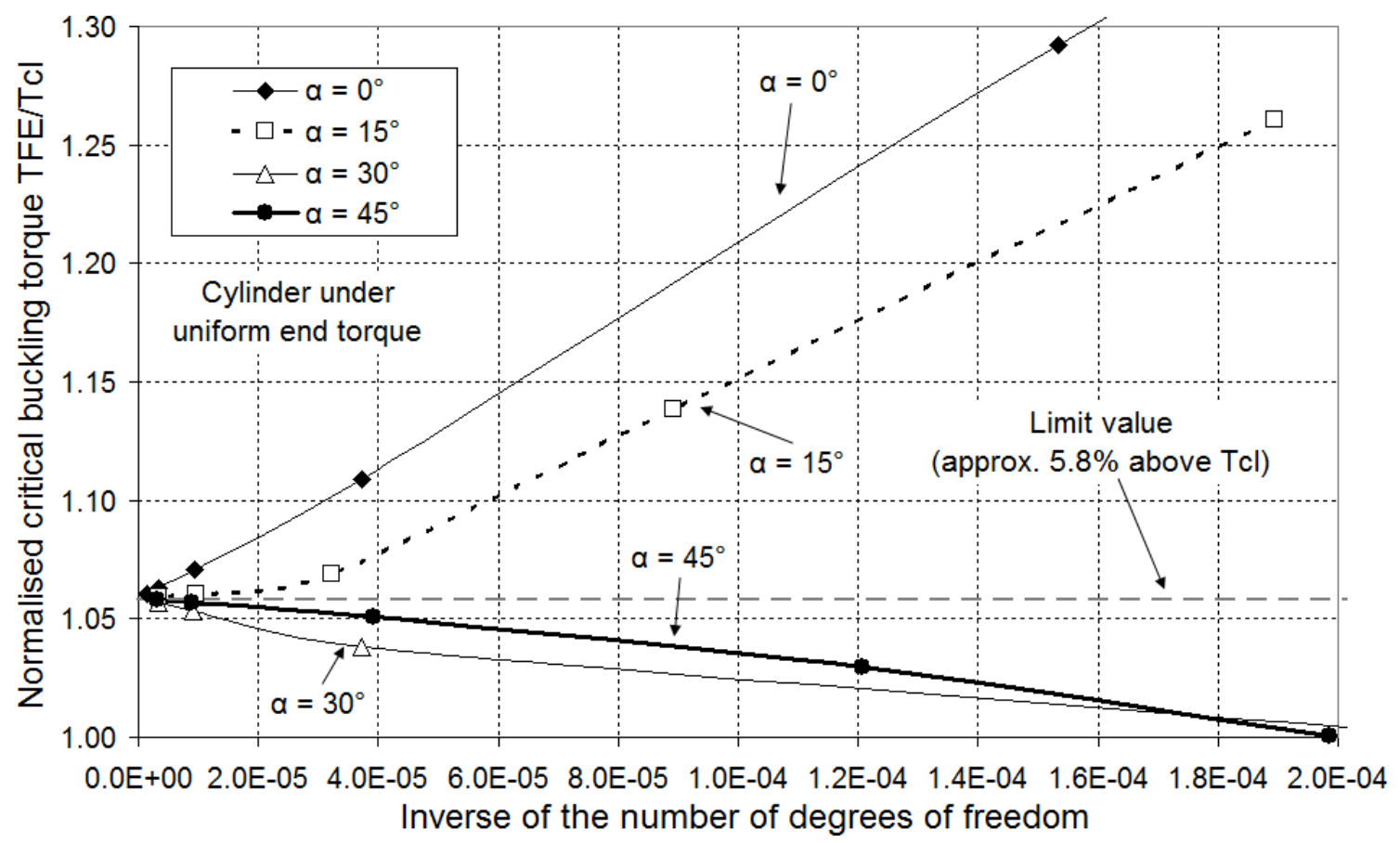

Fig. 15 - Mesh convergence with inverse of dofs for the cylinder under uniform end torque 
Published in: Finite Elements in Analysis and Design, 73C, 42-54.

DOI: http://dx.doi.org/10.1016/j.finel.2013.05.004
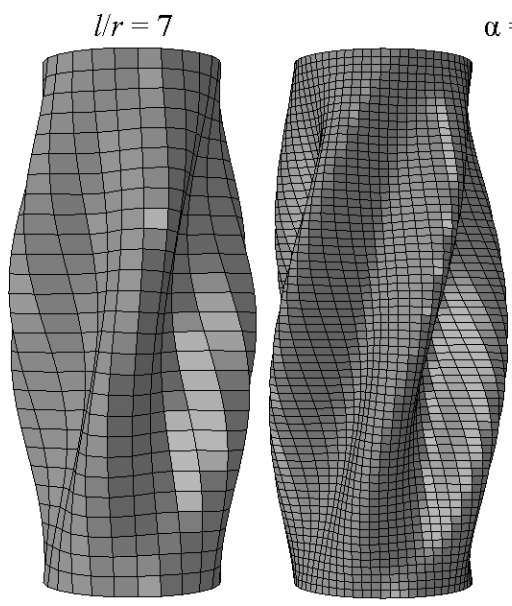

$\alpha=0^{\circ}$
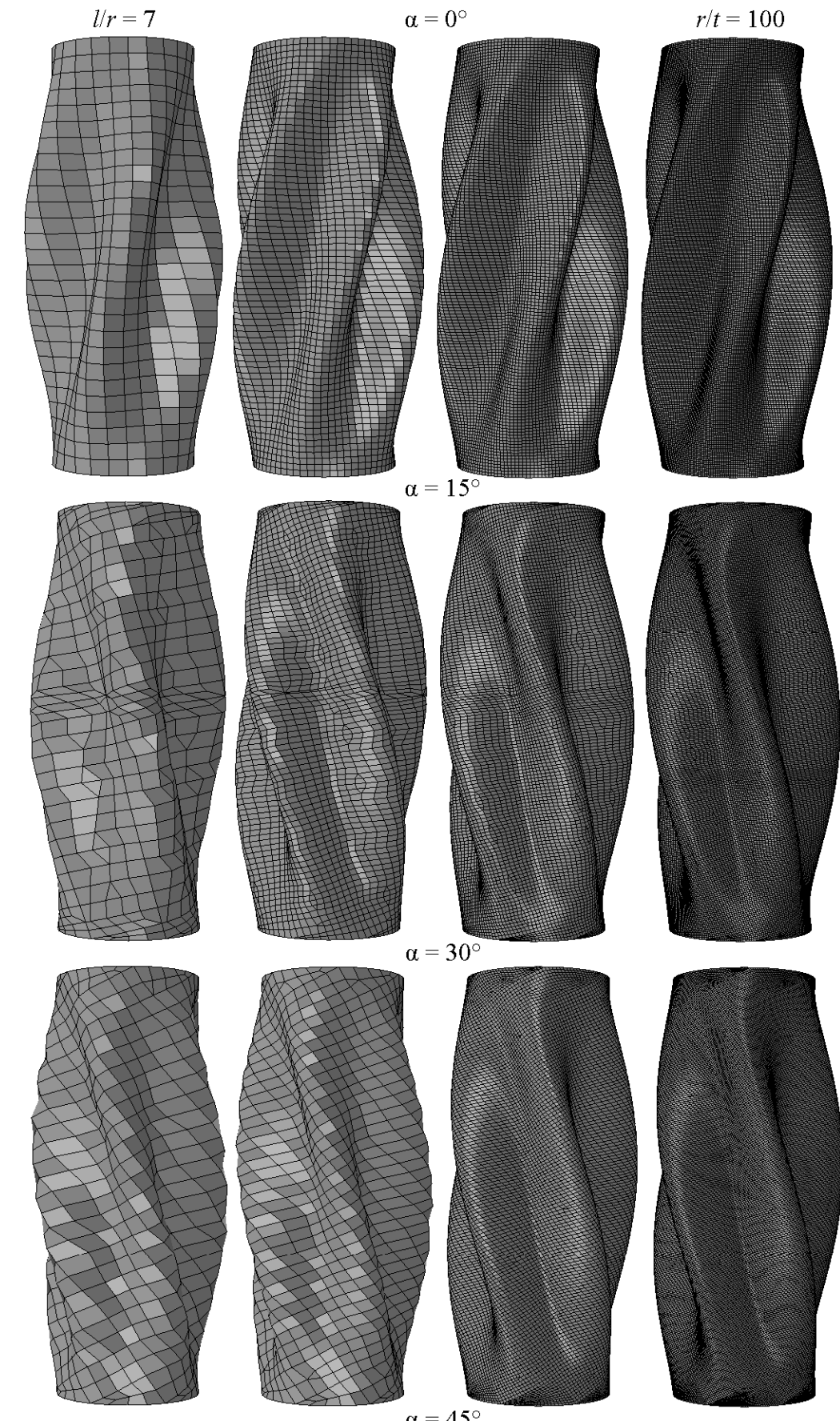

$\alpha=30$
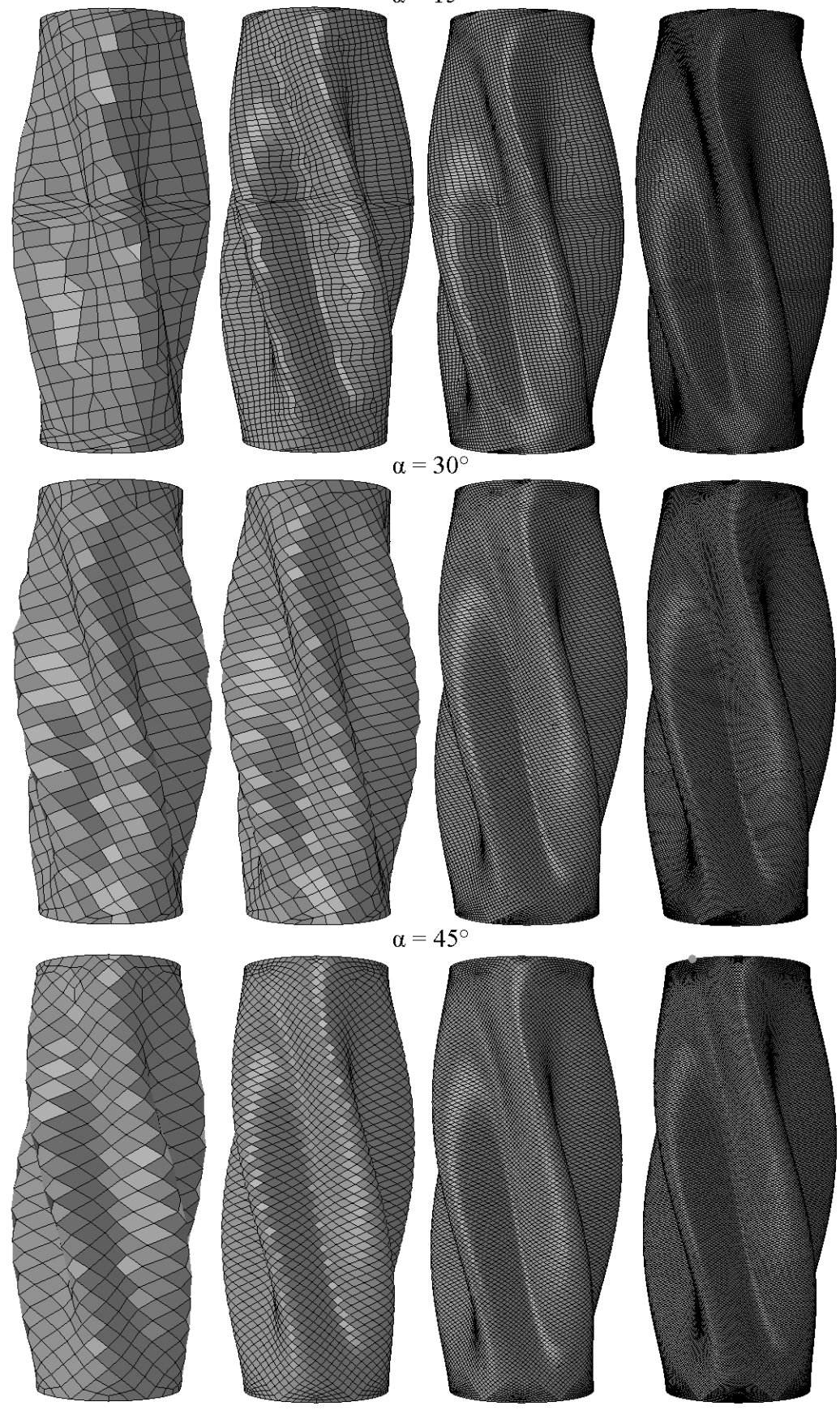

Fig. 16 - Computed linear bifurcation modes for the cylinder under uniform end torque 
Published in: Finite Elements in Analysis and Design, 73C, 42-54.

DOI: http://dx.doi.org/10.1016/j.finel.2013.05.004

The results of the mesh convergence analyses and corresponding linear bifurcation modes are illustrated in Figs 14, 15 and 16 respectively in a manner similar to the previous benchmark test. The analyses converge to a critical value of torque approximately $5.8 \%$ higher than that given by the analytical formula (Eq. 8), an error whose order of magnitude was acknowledged by the authors of that equation [32]. The mesh with $\alpha=45^{\circ}$ exhibits by far the fastest convergence in terms of degrees of freedom because the elements are oriented optimally with respect to the global membrane shear introduced into the shell by the end torque. By contrast, the 'default' regular mesh with $\alpha=0^{\circ}$ exhibits the poorest performance because the elements themselves are now directly under shear. In fact, the regular rectangular mesh requires $532 \%$ more dofs to achieve a $1 \%$ error with the limit value than the inclined mesh with $\alpha=45^{\circ}$. Further, the system exhibits similarly contrasting convergence properties ('from above' vs. 'from below') for the same angles of inclination as the cylinder under axial compression, suggesting that this phenomenon persists for different load cases and is dependent on the angle of inclination of the mesh but apparently not on its relative orientation with respect to the principal axes of stress. Lastly, it may be seen from the critical buckling modes in Fig. 16 that the correct mode is captured by even the coarsest meshes regardless of angle of inclination, a reflection of the imperfection insensitivity of the cylinder under this load condition [35].

\section{Conclusions}

1) This paper outlines a procedure to generate well-structured inclined quadrilateral finite element meshes for the stress and buckling analyses of rectangular plate and cylindrical shell structures.

2) The meshing procedure may be generalised to other types of structures and to analogous problems in other engineering disciplines. It may be readily applied using any finite element pre-processor which supports an external scripting functionality. 
Published in: Finite Elements in Analysis and Design, 73C, 42-54.

DOI: http://dx.doi.org/10.1016/j.finel.2013.05.004

3) A set of mesh convergence studies on four benchmark tests with known analytical solutions was performed to illustrate that a regular orthogonal finite element mesh composed of quadrilateral shell elements performs quite differently depending on its orientation relative to the principal axes of stress in the structure.

4) It was shown that a regular orthogonal finite element mesh may possess a global orientation that is optimal with respect to the principal axes of stress. An optimallyoriented mesh requires significantly fewer elements to achieve a desired accuracy in predicting the correct buckling load and mode.

5) In particular, it was shown that first-order quadrilateral shell elements perform particularly poorly when placed in a configuration that subjects them to pure shear. Depending on the geometry of the structure and on the loading, the default mesh orientation may actually be the worst.

\section{Acknowledgements}

This work was carried out as part of the EU Combitube research project funded by the European Commission, grant number RFSR-CT-2011-00034.

\section{References}

[1] Teng J.G. \& Song C.Y. (2001). "Numerical models for nonlinear analysis of elastic shells with eigenmode-affine imperfections" Int. Jrnl. of Solids and Structures, 38, 3263-3280.

[2] Rotter J.M. (2004). "Buckling of cylindrical shells under axial compression" Chapters and 2 in Buckling of Thin Metal Shells, Eds J.G. Teng \& J.M. Rotter, Spon, London, 1-87.

[3] Song C.Y., Teng J.G. \& Rotter J.M. (2004). "Imperfection sensitivity of thin elastic cylindrical shells subject to partial axial compression". Int. Jrnl. of Solids and Structures, 41, 7155-7180. 
Published in: Finite Elements in Analysis and Design, 73C, 42-54.

DOI: http://dx.doi.org/10.1016/j.finel.2013.05.004

[4] Cai M., Holst J.M.F.G. \& Rotter J.M. (2002a). "Buckling strength of thin cylindrical shells under localised axial compression" 15th ASCE Engineering Mechanics Conference, Columbia University, New York, USA.

[5] Cai M., Holst J.M.F.G. \& Rotter J.M. (2002b). "Buckling of cylindrical tank shells under local axial compression stresses" Int. Conf. on Design, Inspection, Maintenance and Operation of Cylindrical Steel Tanks and Pipelines, ECCS, Prague, Czech Republic, 70-76.

[6] Cai M., Holst J.M.F.G. \& Rotter J.M. (2003). "Parametric study on the buckling of thin steel cylindrical shells under elevated axial compression stresses" 16th ASCE Engineering Mechanics Conference, University of Washington, Seattle.

[7] Sadowski A.J. \& Rotter J.M. (2012). "Slender thin cylindrical shells under unsymmetrical strip loads" Thin-Walled Structures, doi: 10.1016/j.tws.2012.03.021.

[8] Sadowski A.J. \& Rotter J.M. (2010). "A study of buckling in steel silos under eccentric discharge flows of stored solids" ASCE Engineering Mechanics, 136(6), 769776.

[9] Sadowski A.J. \& Rotter J.M. (2011a). "Membrane theory treatment of eccentric flows in concentric hoppers" Thin-Walled Structures, 49(7), 902-912.

[10] Sadowski A.J. \& Rotter J.M. (2011b). "Steel silos with different aspect ratios: II behaviour under eccentric discharge" Jrnl. of Constructional Steel Research, 67(10), $1545-1553$.

[11] Sadowski A.J. \& Rotter J.M. (2011c). "Buckling of very slender metal silos under eccentric discharge" Engineering Structures, 33(4), 1187-1194.

[12] Greiner R. \& Derler P. (1995). "Effect of imperfections on wind-loaded cylindrical shells" Thin-Walled Structures, 23, 271-281. 
Published in: Finite Elements in Analysis and Design, 73C, 42-54.

DOI: http://dx.doi.org/10.1016/j.finel.2013.05.004

[13] Chen L. \& Rotter J.M. (2012). "Buckling of anchored cylindrical shells of uniform thickness under wind load" Engineering Structures, 41, 199-208.

[14] Doerich C. \& J.M. Rotter (2008). "Behaviour of cylindrical steel shells supported on local brackets" ASCE Structural Engineering, 134(8), 1269-1277.

[15] Holst J.M.F.G. \& Rotter J.M. (2005). "Axially compressed cylindrical shells with local settlement" Thin-Walled Structures, 43, 811-825.

[16] Jullien J.F. \& Limam A. (1998). "Effects of openings of the buckling of cylindrical shells under axial compression" Thin-Walled Structures, 31, 187-202.

[17] Michel G., Combescure A. \& Jullien J.F. (2000a). "Finite element simulation of dynamic buckling of cylinders subject to periodic shear" Thin-Walled Structures, 36(2), 111-135.

[18] Michel G., Limam A. \& Jullien J.F. (2000b). "Buckling of cylindrical shells under static and dynamic shear loading" Engineering Structures, 22, 535-543.

[19] Schmidt H. \& Winterstetter Th. A. (1999). "Buckling interaction strength of cylindrical steel shells under axial compression and torsion" Proc. 2nd Int. Conf. on Adv. in Steel Structures, Hong Kong, China, 15-17 December.

[20] Winterstetter Th. A. \& Schmidt H. (2002). "Stability of circular cylindrical steel shells under combined loading" Thin-Walled Structures, 40(10), 893-910.

[21] Iliescu T. (1999). "A flow-aligning algorithm for convection-dominated problems." International Journal for Numerical Methods in Engineering, 46, 9931000.

[22] Frey P.J. \& Alauzet F. (2005). "Anisotropic mesh adaptation for CFD computations." Computer methods in applied mechanics and engineering, 194, 50685082. 
Published in: Finite Elements in Analysis and Design, 73C, 42-54.

DOI: http://dx.doi.org/10.1016/j.finel.2013.05.004

[23] Li X., Shephard M.S. \& Beall M.W. (2005). "3D anisotropic mesh adaptation by mesh modification." Computer methods in applied mechanics and engineering, 194, 4915-4950.

[24] D'Azevedo E.F. (2000). "Are bilinear quadrilaterals better than linear triangles?" SIAM J. Sci. Comput., 22(1), 198-217.

[25] Troyani N., A Pérez \& P. Baíz (2005). "Effect of finite element mesh orientation on solution accuracy for torsional problems." Finite Elements in Analysis and Design, 41, 1377-1383.

[26] Hamide M., Massoni E. \& Bellet M. (2008). "Adaptive mesh technique for thermal-metallurgical numerical simulation of arc welding processes." International Journal for Numerical Methods in Engineering, 73, 624-641.

[27] El khaoulani R. \& Bouchard P.O. (2012). "An anisotropic mesh adaptation strategy for damage and failure in ductile materials." Finite Elements in Analysis and Design, 59, 1-10.

[28] George P-L. \& Borouchaki H. (1998). "Delaunay Triangulation and Meshing Application to Finite Elements" Editions Hermes, Paris.

[29] Zienkiewicz O.C., Taylor R.L. \& Zhu J.Z. (2006). "The finite element method It's basis and fundamentals". 6th edition, Elsevier.

[30] Zienkiewicz O.C. \& Taylor R.L. (2005) "The finite element method - For solid and structural mechanics". 6th edition, Elsevier.

[31] ABAQUS (2011). “ABAQUS Version 6.10.1 Theory Manual” Dassault Systèmes Simulia Corp., Providence, RI, USA.

[32] Timoshenko S.P. \& Gere J.M. (1963). "Theory of elastic stability" McGraw-Hill International Editions. 
Published in: Finite Elements in Analysis and Design, 73C, 42-54.

DOI: http://dx.doi.org/10.1016/j.finel.2013.05.004

[33] Brush D.O. \& Almroth B.O. (1975). "Buckling of bars, plates and shells". McGraw-Hill.

[34] Cook R.D., Malkus D.S., Plesha M.E. \& Witt R.J. (2002). "Concepts and applications of finite element analysis" 4th edition. John-Wiley \& Sons.

[35] Yamaki N. (1984). "Elastic stability of circular cylindrical shells" North-Holland, Amsterdam.

[36] Donnell L.H. (1933). "Stability of thin tubes under torsion" NACA Report No. 479. 